\title{
A REVIEW ON SPECTRUM SENSING METHODS FOR COGNITIVE RADIO NETWORKS
}

\author{
Mr. K.Kaarthik, Mr.P.T.Sivagurunathan, Mrs.S.Sivaranjani, \\ ${ }^{1,2,3}$ Assistant Professor, Dept of ECE, M.Kumarasamy college of engineering, Karur. \\ E-mail: ${ }^{1}$ kaarthikk.ece@mkce.ac.in, ${ }^{2}$ sivaphd2017@gmail.com ${ }^{3}$ Swame12@gmail.com
}

\begin{abstract}
In Wireless Communication, Radio Spectrum is doing a vital role; for the future need it should use efficient. The existing system, it is not possible to use it efficiently where the allocation of spectrum is done based on fixed spectrum access (FSA) policy. Several surveys prove that it show the way to inefficient use of spectrum. An innovative technique is needed for spectrum utilization effectively. Using Dynamic spectrum access (DSA) policy, available spectrum can be exploited. Cognitive radio arises to be an attractive solution which introduces opportunistic usage of the frequency bands that are not commonly occupied by licensed users. Cognitive radios promote open spectrum allocation which is a clear departure from habitual command and control allocation process for radio spectrum usage. In short, it permits the formation of "infrastructure-less" joint network clusters which is called Cognitive Radio Networks (CRN). Conversely the spectrum sensing techniques are needed to detect free spectrum. In this paper, different spectrum sensing techniques are analyzed.
\end{abstract}

Keywords- Sensing Techniques, Spectrum, Cognitive Radio, Detection

\section{INTRODUCTION}

The challenge of wireless network design is the use of frequency spectrum. With the drastic increase in communication applications in various countries, almost the entire spectrum has been fully allocated resulting in the spectrum congestion problem. From the latest report of Federal Communication Commission (FCC) about 15 to $85 \%$ of spectrum is not utilized [1]. Usage of the frequency band can be enhanced by introducing Secondary User (SU) which will continuously identify the presence of PU called the spectrum sensing, and utilize licensed band when Primary User (PU) is absent. Secondary User (SU) will transmit their knowledge without disrupting licensed user (PU), such an opportunity is called spectrum hole and the spectrum device that Indentify these holes are called Cognitive Radio (CR). Cognitive radio is widely as one of the Hopeful technology for the future wireless communication. According to Federal Communication Commission

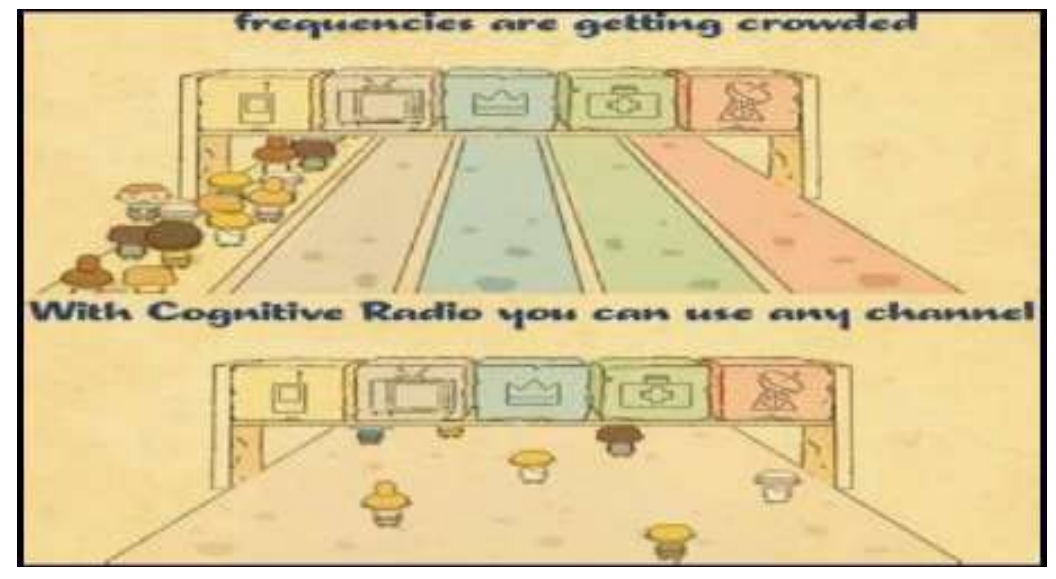

Figure 1. Frequency utilization in $\mathrm{CR}$

\section{SPECTRUM SENSING}

Spectrum sensing or PU detection is an major function for cognitive radio. It can be defined as process of detecting spectrum holes by sensing radio spectrum all around cognitive radio receiver. Temporary/ provisional usage of unused frequency bands by CR is commonly known as spectrum holes. Spectrum holes are of two types, temporal spectrum holes and spatial spectral holes.

A spectrum hole which is unoccupied by the PU during the processtime of sensing is temporal spectrum hole, hence can be used by the SU at that time slot. Band which is not occupied by PU at some spatial areas is spatial spectrum hole, therefore it can be engaged by SU as well as outside of this area. CR hops to another type of spectrum holes if the band is used more by PU as shown in figure. 2 


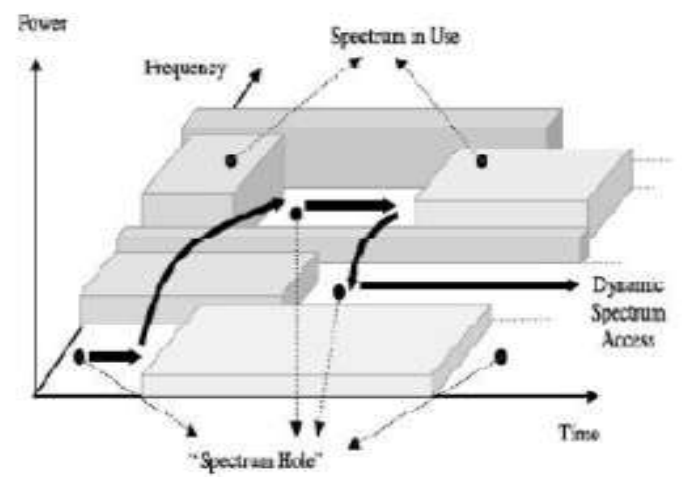

Figure 2. Spectrum holes

\section{SPECTRUM SENSING TECHNIQUES}

The fundamental principle for spectrum sensing is shown in the figure 3 , a licensed Tx sends records to its required $R x$ in a certain licensed spectrum band. A couple of CR users (CR Tx and CR Rx) intends to contact the spectrum holes for minor communication. [2-3] To assurance the protection of PUs, the CR Tx needs to execute spectrum sensing to find spectrum holes. In specific, the CR Tx is necessary to detect whether there is an active primary Rx within the coverage of the CR Tx. If not, the CR Tx can safely spread to the CR Rx using the identified spectrum hole [2-3]. Otherwise, the CR users are not permitted to use the band. Therefore, detecting the nearby primary Rx's can straightly identify the spectrum hole, which is called as the direct spectrum sensing. [4].

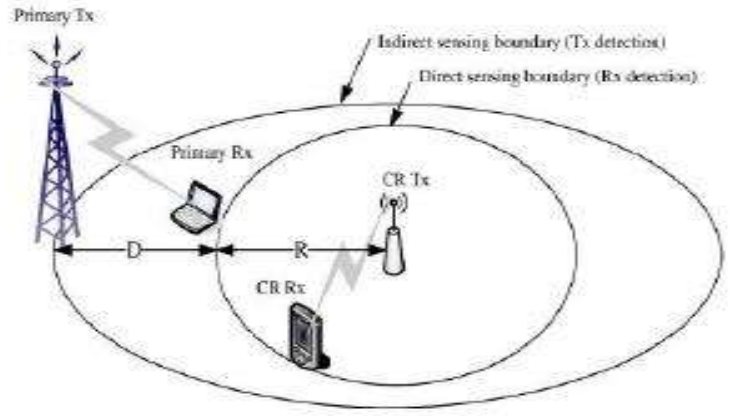

Figure 3. Principal of channel sensing

\subsection{Classification of Spectrum Sensing Methods}

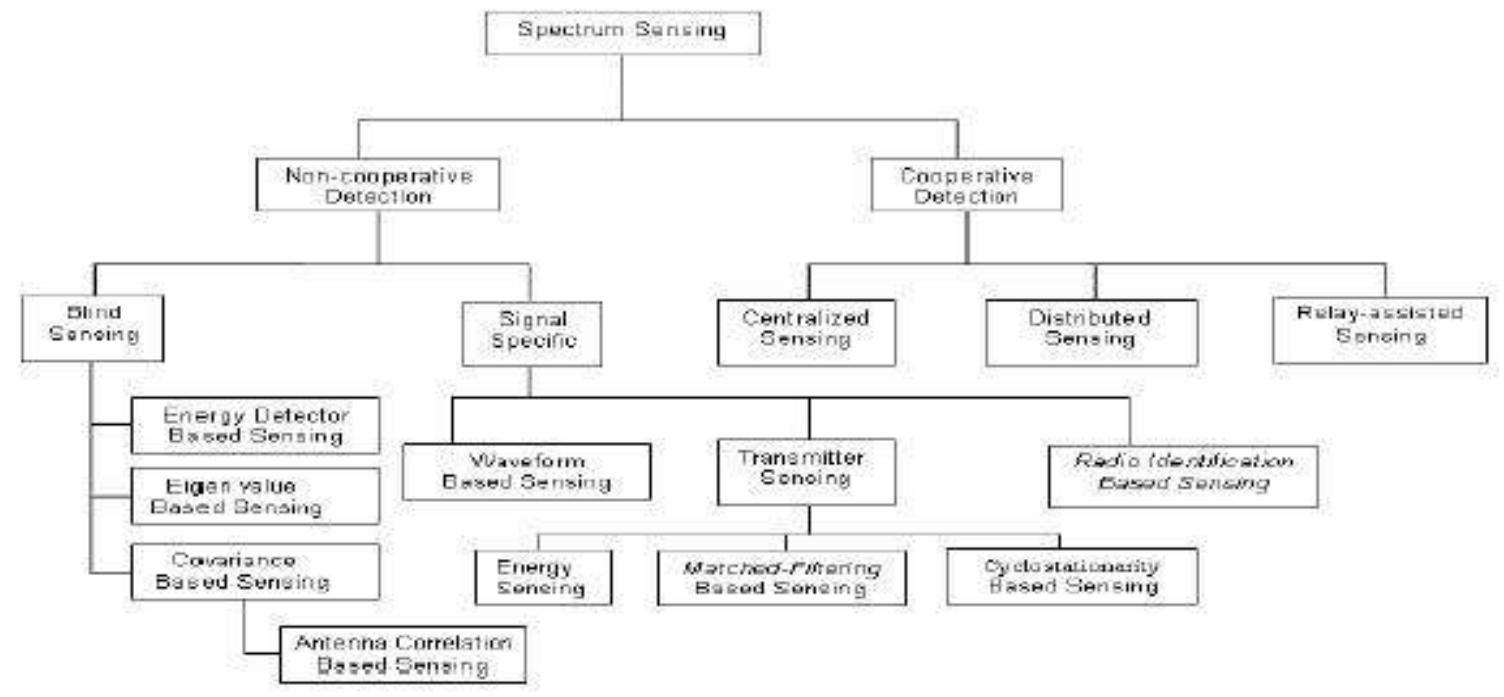

Figure 4. Different Schemes of Spectrum Sensing 
We can broadly divide spectrum sensing techniques under two categories: Cooperative Detection Technique and Noncooperative Detection Technique.

\subsubsection{COOPERATIVE DETECTION TECHNIQUE}

In this technique group of CR's split sensing information so as to get a more well-organized result. In this process group of secondary user (Su) gather the information regarding channel possession and sustain this information into spectrum map Focused by bit-vector. Su periodically transmit it to the Central Coordinator as part of control message. Central coordinator takes the bitwise-OR of spectrum maps, to decide the set of UHF channels accessible at all of the nodes. After that controller select the best accessible channel and broadcast it back to SU. This technique exploit the spatial diversity inherent to a multi-user network. It can be accomplished in a federal or spread fashion. [5].There are generally three approaches for cooperative spectrum sensing: Centralized approach, Distributed approach, Relay-assisted cooperative.

\section{Generally two basic steps are involved in the cooperative sensing as:}

Detection of PU: In this segment of cooperative sensing all cognitive users' attempt to locate out the chief vacant channel.

Reporting: In this the results of result phase is reported back to the FC. There is a big question in the information phase that is when CUs send their initial results to FC there may be a obstruction with the PUs. To struggle it selective relay based cooperative scheme came into the survival in which all CUs can send their detection results to FC without using a devoted channel depending on a selective algorithm based on if nonexistence of PU is detected or not.

If $\mathrm{K}_{\mathrm{th}} \mathrm{CU}$ detects nonexistence of $\mathrm{PU}$ in its phase of detection then it starts to transmit a cyclic redundancy check (CRC)coded representing signal to the $F C$ over the $L_{\text {th }}$ orthogonal sub-channel of the primary channel, else nothing is transmitted as of CU to avoid interfering with PU. On other side of FC, if the CRC examination is successful on the $L_{\text {th }}$ orthogonal subchannel, FC consider the lack of PU as the initial result detected by CU if not, it considers the attendance of $\mathrm{PU}$ as the CU initial finding result [6-7].

\subsubsection{NON CORPORATIVE DETECTION TECHNIQUE}

In Non corporative detection method individual radios works close by and separately to carry out their own discovery of unused frequency band and habitation of spectrum [8]. Three methods have been discussed in the following subsections under non cooperative detection.

\subsubsection{Blind Sensing}

In this approach to cognitive radio cooperative spectrum sensing, there is a node called fusion center (FC) controls inside the system that collect the sensing information from all the sense nodes or radios inside the network. It then analysis the information and determine the frequencies which can be used.

\subsection{Energy Detector based sensing}

Energy detection is a method to detect signal using an energy detector (also known as radiometer) to identify the deficiency or attendance of signal in the band. Energy detector based technique is universal way of sensing the spectrum because of its low computational time and performance complexities [9]. It is very simple and practical method as receiver does not need any information about PU's signal to be detected, so it is broadly adopted. The signal is recognized by evaluate the threshold which depends on the noise floor with the output of energy detector [10] However there are some limitations of this method which includes incapability to differentiate interference between signal from a user signal and noise, it is also not efficient for those signals whose signal power has been spread over a wideband.

\section{However, there are some drawbacks for energy detection:}

The result threshold is subject to varying signal to -noise ratios (SNR's).

It cannot differentiate interference from a user signal.

It is not successful for signals whose signal power has been expand over a wideband.

\subsection{Eigen value based Sensing}

The Eigen value of the covariance matrix of the received signal can also give out the reason of primary detection. With the assist of random matrix theory, the relation of the maximum eigen value to the minimum eigen value is quantized, and one of the quantized values is selected as detection threshold

\subsection{Covariance based Sensing}

As a substance of fact statistical covariance matrices of the received signal and that of noise are usually unlike. By operate this diversity we can distinguish the desired signal component from locale noise.

\subsubsection{Signal Specific}


This sensing method requires prior information of Primary User (PU) signal.

\subsection{Waveform based Sensing}

This method is only appropriate to systems with known signal patterns which could be introduction, midambles, frequently transmitted pilot patterns, scattering sequences and etc. Preamble is a known sequence transmitted before each explode and Midamble is transmitted in the center of a burst or slot. It is also known as waveform-based sensing or coherent sensing. It is shown that waveform based sensing Outperforms energy detector based sensing in dependability and convergence time. It is shown that the appearance of the sensing algorithm increases as the distance of the known signal sketch increases. And in the attendance of a known pattern, sensing can be performed by correlating the traditional signal with a known copy of itself.

\subsection{Transmitter Based Sensing}

Here, the cognitive radio try to discern areas of used or idle spectrum by formative if a prime user is transmitting in its vicinity. This approach is predicated on detecting not the strongest transmitted signal from a prime user, but the weakest. The design is that the weakest signal create primary transmitter would preferably be the one farthest away from the cognitive radio, but still subject to RF interference from the radio. The three main finding techniques which rely on this premise for transmitter detection are described below.

\subsection{1 Energy Sensing}

If a receiver cannot get together enough information about the primary user's signal, such as in the case that only the power of random Gaussian noise is known to the receiver, the most favorable detector is an energy detector. Energy detection is easy and can be implemented capably by using a Fast Fourier Transform (FFT) algorithm[15].

\subsection{Matched Filter Sensing}

The coordinated filter works by associate a known signal, or pattern, with an unknown signal to detect the occurrence of the pattern in the unknown signal. Because most wireless network systems have pilots, preamble, synchronization word, or scattering codes, these can be used for coherent (matched filter) detection. A big bonus in favor of the harmonized filter is that it requires less time to attain a high dispensation gain due to coherency.

The main shortcomings of the matched filter are:

- It requires a priori information of the primary user signal which in a factual world circumstances may not be accessible.

- an additional drawback of matched filter sensing is that cognitive radio requires a devoted receiver for licensed user.

\subsection{Cyclostationary based sensing}

Cyclostationary detector utilizes the Cyclostationary characteristic of the signals for spectrum sensing. If mean and autocorrelation of Cyclostationary are a cyclic function then a signal is supposed to be Cyclostationary. [11]. aspect detection refers to sunder out the features from the received signal and execute detection based on the extracted feature [12-13]. Cyclostationary finding is superior option than energy detector in case where energy detection is not so efficient. It performs improved than energy detector because of its noise refusal ability as noise is totally random and does not show any episodic behavior.

Cyclostationary detector can distinguish noise from approved user (PU) signal and can also be used for detecting fragile signal at a very low signal-to-noise share region. Cyclostationary detection method is best used when we have no earlier knowledge about approved user signal. The disadvantage of this method is the complexity of result and long sensing time.

\subsubsection{Radio Identification Based Sensing}

This process veers from the characteristic study of interference which is generally transmitter-centric. normally, a transmitter control its intervention by regulating its output transmission power, its out-of-band emissions, based on its locality with admiration to other users. Cognitive radio identification-based detection concentrates on measuring intervention at the receiver. [14]

\section{Conclusion}

In this paper offered the pros and cons of different spectrum sensing technique, and execute the assessment in terms of process, accuracies and difficulty. There exist number of issues to be addressed in terms of primary signal finding time, hardware supplies and computational difficulty and also survey provides a solution to some general problems in spectrum sensing. The graph in figure 5 shows different sensing process in terms of their sensing exactness and complexity. Cognitive radio technology will be applied to many real systems in the prospect and can be used as base paper by many aspirants, who wish to work in this field. 


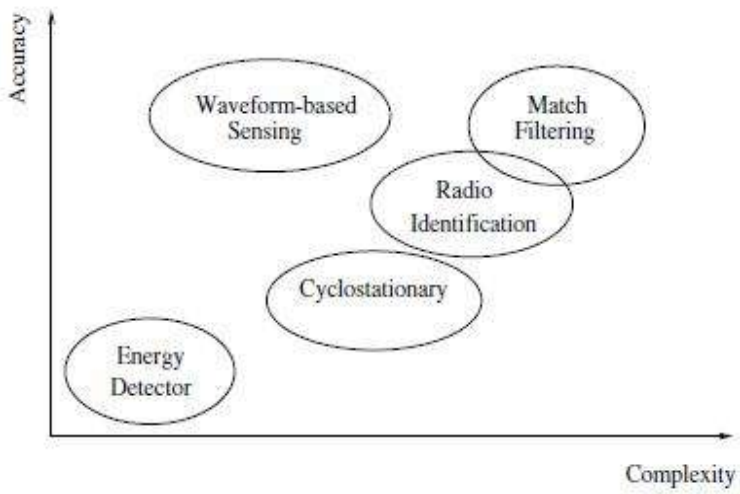

Figure 5. Main sensing methods in terms of their sensing accuracies and complexities.

\section{Acknowledgment}

Our thanks to M.Kumarasamy college of Engineering for offering us the opportunity to do this wonderful Survey, and to Dr. V. Kavitha, Principal, Dr. K.Sundararaju, Dean/ EE and The HOD Prof. A. Sridevi whose contribution in stimulating suggestions and encouragement, helped us to coordinate our project, especially in writing this paper.

\section{Reference}

1. Federal communications commission(FCC), spectrum policy task force, ET Docket no.2, pp.1-135, 2002

2. W.-Y. Lee and I. F. Akyildiz, "Optimal spectrum sensing framework for cognitive radio," IEEE Trans. Wireless Commun., vol. 7, no.10, Oct.2008, pp. 3845-3857.

3. S. Keshavamurthy and K. Chandra, "Multiplexing analysis for dynamic spectrum access," in Proc. IEEE MILCOM'06, Washington, DC, USA,Oct. 2006

4. Z. Ji and K. J. R. Liu, "Dynamic spectrum sharing: a game theoretical overview," IEEE Commun. Mag., vol. 45, no. 5, pp. 88-94, May 2007.

5. A.D.cabric, s. mishra, r. brodersen, "Implementation issues in spectrum sensing for cognitive radios", in: proc. Of asilomar conf. on signals, systems, and computers, vol. 1, 2004, pp. 772-776.

6. Ghurumuruhan ganesan and ye (geoffrey) li "Cooperative spectrum sensing in cognitive radio, part ii: multiuser networks" ieee transactions on wireless communications, vol. 6, no. 6, june 2007

7. G. Ganesan and Y. (G.) Li, "Cooperative Spectrum Sensing in Cognitive Radio - Part I: Two user networks," accepted for publication to IEEE Trans. on Wireless Commun.

8. W. Zhang, K. Letaief, - "Cooperative spectrum sensing with transmit and relay diversity in cognitive radio networks - [transaction letters]",IEEE Transactions on Wireless Communications, vol.7, No.12, pp. 4761-4766 2008.

9. Cabric, D.; Mishra, S.M.; Brodersen, R.W., "Implementation issues in spectrum sensing for cognitive radios," in Signals, Systems and Computers, 2004. Conference Record of the Thirty-Eighth Asilomar Conference on , vol.1, pp.772-776 , 7-10 Nov. 2004

10. Tevfik $Y "$ ucek et al, "A Survey of Spectrum Sensing Algorithms for Cognitive Radio Applications", ieee communications surveys \& tutorials, vol. 11, No. 1, first quarter, 2009.

11. Tevfik y"ucek and h"useyin arslan "a survey of spectrum sensing algorithms for cognitive radio applications" ieee communications surveys \& tutorials, vol. 11, no. 1, first quarter 2009

12. T.ulino and s. verdú, random matrix theory and wireless communications. delft, the netherlands: now, 2004

13. Y. zeng and y. liang, "maximum-minimum eigenvalue detection for cognitive radio," in proc. ieee 18th int. symp. personal, indoor mobile radio commun. (pimrc), 2007, pp.1-5.

14. Z. han,rongfei fan, and hai jiang, "Replacement of spectrum sensing in cognitive radio" ieee transactions on wireless communications, vol. 8, no. 6, june 2009

15. V.Kavitha, C.Gayathri "An Analysis on Routing and Issues in Network Layer in WMN's" International Journal of Scientific and Engineering Research, vol.6, june 2015. 